\title{
Chemotherapeutic agents for the treatment of hepatocellular carcinoma: efficacy and mode of action
}

\author{
Saad Shaaban, ${ }^{1}$ Amr Negm, ${ }^{2}$ Elsayed E. Ibrahim, ${ }^{3}$ Ahmed A. Elrazak ${ }^{4}$ \\ ${ }^{1}$ Department of Chemistry; ${ }^{2}$ Department of Biochemistry; ${ }^{3}$ Department of Zoology; ${ }^{4}$ Department \\ of Botany, Mansoura University, Egypt
}

\begin{abstract}
Hepatocellular carcinoma (HCC) is a dreaded malignancy that every year causes half a million deaths worldwide. Being an aggressive cancer, its incidence exceeds 700,000 new cases per year worldwide with a median survival of 6-8 months. Despite advances in prognosis and early detection, effective HCC chemoprevention or treatment strategies are still lacking, therefore its dismal survival rate remains largely unchanged. This review will characterize currently available chemotherapeutic drugs used in the treatment of HCC. The respective mode(s) of action, side effects and recommendations will be also described for each drug.
\end{abstract}

\section{Introduction}

Cancer is the leading cause of death in developed countries and the second leading cause of death in developing countries. Liver cancer is one of the most frequent and dismal malignancies.

Correspondence: Amr Negm, Department of Biochemistry, Faculty of Science, Mansoura University, El-Gomhorya Street, 35516 Mansoura, Egypt. Tel.: 0020.1018933660 - Fax: 0020.502.397900.

E-mail: amrbiochem@googlemail.com ; amrnigm@mans.edu.eg

Key words: liver cancer, hepatocellular carcinoma, molecular therapies, chemoresistance.

Contributions: the authors contributed equally to this work.

Conflict of interests: the authors declare no potential no conflict of interests.

Acknowledgements: the authors wish to express their sincere thanks to the staff members of the Faculty of Science, Mansoura University, Egypt for the facilities and support provided.

Received for publication: 4 February 2014.

Accepted for publication: 6 March 2014.

This work is licensed under a Creative Commons Attribution NonCommercial 3.0 License (CC BY-NC 3.0).

(C) Copyright S. Shaaban et al., 2014

Licensee PAGEPress, Italy

Oncology Reviews 2014; 8:246

doi:10.4081/oncol.2014.246
Hepatocellular carcinoma (HCC) is the third most common cause of cancer death, the most recurrent primary liver cancer and the fifth most common malignancy worldwide with over 700,000 new cases per year. Although the incidence of HCC is steadily rising worldwide, $25 \%$ of patients only benefit from the curative treatment. ${ }^{1}$ Furthermore, most patients seek treatment when the disease is beyond curative treatment (surgery or percutaneous ablation), and palliative care is the only alternative. ${ }^{2,3}$

The treatment of patients with HCC presents a major challenge, because associated cirrhosis limits the choice of chemotherapeutic agents. The possible therapeutic options fall into five main categories: i) surgery, including tumor resection and liver transplantation; ii) percutaneous interventions, including ethanol injection and radiofrequency thermal ablation; iii) transarterial interventions, including embolization and chemoembolization; iv) radiation therapy and v) gene and immune therapeutic drugs. ${ }^{4}$

Although treatment options have become more diverse in recent years, improvements in HCC survival rates lag far behind those achieved in other tumors. Until recently, no real therapy existed for patients with advanced HCC. Systemic chemotherapy in particular has been disappointing, not only because of the chemoresistance of HCC, but also of major side effects which make them poorly tolerated by patients with liver cirrhosis. ${ }^{4}$ The management of HCC is dictated by the degree of underlying liver dysfunction, the burden of malignancy and the patient's performance status. In contrast to the early-stage HCC, there are limited treatment options for advanced HCC. In this context, several therapeutic agents have been developed over the past 50 years in order to provide a better response and improve the survival of HCC patients. ${ }^{5-7}$ Moreover, different modulation strategies and administration routes have been proposed to enhance the antitumor activity of these agents. -11 $^{8-1}$

As the treatment of patients with HCC presents a major challenge, herein we report a systematic review of the literature on state-of-theart chemotherapeutic drugs which have been used in several clinical trials on the treatment of HCC. In this review, we focus on how these agents exert their effect in order to gain a better understanding of the efficacy and safety of currently available drugs.

\section{Systemic therapy of advanced hepatocellular} carcinoma

\section{Hormonal therapy}

\section{Octreotide}

Octreotide, somatostatin (SST) hormone mimic, exhibits regulatory and suppressive effects against several adenocarcinomas including 
breast, kidney, colon and ovary carcinomas. ${ }^{12,13}$ A few years ago SST analogues emerged as a promising treatment for nonsurgical HCC, but the effectiveness of this approach is still a controversial topic. ${ }^{14}$

Octreotide was firstly used for HCC treatment by Kouroumalis et al. in a randomized trial with 58 patients. ${ }^{15,16}$ In this study, octreotide appeared to be a promising drug by doubling the survival in this group. ${ }^{16} \mathrm{~A}$ similar but not randomized study with long-acting analogues from the same group confirmed the results using historical controls. ${ }^{15}$ In these two reports, it was clearly demonstrated that differences in survival are mostly apparent after 6 months of treatment. This is considered to be a reasonable result, since SST is not a rescue drug and time is required before any benefit can be achieved. Moreover, in these two reports an impressive improvement in the quality of life was noticed, and even patients with an advanced disease retained their appetite, a reasonable body weight, and sense of well-being until the very end. Since then, several clinical studies were published and the role of SST receptors (SSTR) became a topic of intensive research and controversy. ${ }^{17}$

Octreotide improves the response of HCC SSTR [+] patients over those of HCC SSTR[-]. The molecular mechanisms of SST antineoplastic activities are therefore attributed to the direct and indirect proliferation suppression caused by SSTR which is over expressed in HCC. ${ }^{15,16}$ Generally, there are at least six main mechanisms by which SST and its analogues inhibit HCC progress: i) inhibition of secretion of growth hormone, insulin and gastrointestinal (GI) hormones (gastrin, glucagon) that could be involved in the regulation of tumor growth; ii) direct or indirect inhibition of insulin-like growth factors (IGF-I and IGF-II) or IGF binding proteins; iii) direct inhibition of angiogenesis (in vivo and in vitro); iv) direct antiproliferative effect on cancer cells via specific SSTR; v) induction of apoptosis (dependent on or independent from p53) and vi) immune modulation antineoplastic effect. ${ }^{12,18,19}$ Nevertheless, the exact function of somatostatins in HCC is still argumentative.

In some cases a poor tumor growth inhibition was observed with SST analogues. From the clinical point of view, there are several possible reasons for this effect: i) SSTR down regulation in tumor cells accompanied by a decrease either in the number and/or the affinity of SSTR due to continued exposure to the agonist (tachyphylaxis); ii) responsiveness decrease due to receptor uncoupling by a second messenger activation (desensitization); iii) non homogenous expression of SSTR in tumors with predominance or emergence of receptor-negative cell clones; iv) upregulation of binding sites that do not recognize the specific analogue and/or escape of cancer cells that do not express SSTR at all; v) mutations in SSTR genes that might lead to the absence of functional SSTR proteins; vi) production of endogenous peptides like cortistatin that affect the expression and internalization of the SSTR; vii) SSTR expression downregulation by pro-inflammatory cytokines, such as the tumor necrosis factor TNF $\alpha$ and interleukin-1 (IL-1). ${ }^{20-22}$ To conclude, octreotide could be used as an effective treatment for various endocrine tumors. Nevertheless, its role in advanced HCC remains controversial.

\section{Tamoxifen}

Tamoxifen (TMX), a triphenylethylene non-steroidal anti-estrogen, is one of the most important hormonal drugs which is being used to treat breast cancer for over a decade. Interestingly, TMX was also used in HCC and showed to prolong survival of some patients in advanced HCC. ${ }^{23,24}$ As the liver is a hormone-sensitive organ, various evidence suggested that sexual hormones and their receptors have a role in liver carcinogenesis. Accordingly, the presence of estrogen receptors (ER) in the liver and the supposed causal relationship between sexual steroids and liver tumors suggested the possibility of using TMX in the treatment of HCC..$^{25,26}$ In this context, cell culture experiments on the human hepatoblastoma cell lines HepG2 and Hep3B showed that TMX induced growth inhibition in a dose-dependent manner at the nanomolar range..$^{27,28}$

Estrogen-positive HCC receptor responds to TMX treatment; however, more than $50 \%$ of HCCs are ER-negative. Interestingly, TMX at higher doses is known to have a therapeutic action, regardless of the ER status. Therefore high-dose TMX would theoretically have a therapeutic action on both ER-positive and ER-negative HCC suggesting ER-independent mechanisms..$^{29,30}$

Mount reports suggested that TMX induces cell death via multifactorial pathways; however the underlying mechanism of TMX is not yet fully understood. In HepG2 cells, it inhibits proliferation via down-regulation of mammalian target of rapamycin activity and survivin expression. Furthermore, TMX is believed to induce apoptosis by blocking anti-apoptotic pathways, such as PI3K-Akt, and mitogen-activated protein kinase, which in turn is involved in cell proliferation and tumorigenesis. ${ }^{27,28}$

The efficacy of TMX therapy in advanced HCC is controversial and conflicting. Recent meta-analysis showed that it did not provide significant anti-tumoral or survival effects. In some cases, a significant trend toward a negative dose response was observed. It was believed that the positive results identified in the early 1990s were due to a methodological bias and random error of small-sized studies. ${ }^{31}$

In the absence of proven therapy for inoperable HCC, clinicians (especially in the third world) are still using TMX on an ad-hoc basis. In the meantime, we do not recommend the use of TMX in patients with HCC, as it did not offer any benefits in terms of survival and is likely to be detrimental.

\section{Biologic and biochemical therapy}

\section{Thalidomide}

Hepatocellular carcinoma (HCC) is a typical hypervascular tumor and is considered to be an antiangiogenic and angiogenesis-dependent tumor. ${ }^{32}$ It was found that angiogenic factors, such as the vascular endothelial growth factor (VEGF), basic fibroblast growth factor (bFGF) and matrix metalloproteinases were over expressed in HCC cells and also in the surrounding stroma cells. ${ }^{33,34}$ Furthermore, elevated serum levels of angiogenic factors were also found in patients with liver cirrhosis. ${ }^{35}$ The high prevalence of HCC-induced angiogenesis made it a logical target for cancer therapy. The antiangiogenic therapy has many advantages over the conventional cytotoxic therapy targeted at cancer cells; firstly, the tumor vasculature is pharmacokinetically more homogeneous than the tumors which could be located in different parts of the body. Secondly, the primary target cells (endothelial cells) are genetically stable diploid cells and acquired drug resistance could be rare. Thirdly, partial damage to the endothelium would be enough to block blood supply to the tumors and result in growth inhibition or even tumor shrinkage. Consequently, the antiangiogenic therapy is a reasonable choice for HCC growth control and inhibition..$^{36,37}$

Thalidomide is a glutamic acid derivative which was firstly introduced in the 1950, as an anti-inflammatory agent. Ten years later, thalidomide was banned due to its teratogenic action which was related to the inhibition of new blood vessel formation. ${ }^{38,39}$ Once more, thalidomide attracted the attention of oncologist due to its effects in certain multiple myelomas. ${ }^{40,41}$

The preliminary efficiency of thalidomide in case of advanced HCC showed only modest responses with acceptable toxicity. ${ }^{35}$ In this context, the efficacy and tolerability of fixed low-dose thalidomide was further studied by Hsu et al. The study showed an overall survival time of 18.7 weeks with an overall response rate of $6.3 \% .{ }^{42}$ On the other hand, a high-dose study by Patt et al. showed an overall response rate of $5 \%$ with 6.8 moths survival time. ${ }^{43}$ 
The potential mechanisms of thalidomide's anticancer activity were attributed to its active four-peptide signals. ${ }^{35}$ It down-regulates IL-6, bFGF, VEGF, and TNF $\alpha$ that are released by tumors in order to stimulate cell growth and neoangiogenesis. Furthermore, it induces the proliferation of CD8-positive T-cells and hence modulates the immune function. Moreover, it suppresses cyclooxygenase- 2 activity and thereby reduces the production of prostaglandin. Additionally, thalidomide modulates several cell adhesion molecules, which are involved in metastases. ${ }^{36,44-46} \mathrm{Up}$ to now, it is unclear which of these four activities are responsible for the thalidomide action.

In conclusion, Thalidomide is considered as an alternative to more expensive molecular targeted therapies with modest responses and acceptable toxicity to HCC. The exact mode(s) of action still requires more clarification, if it is intended to be used as a single antitumor drug or in combination with other supportive drugs. Of course there are side effects, such as central and peripheral neurologic toxicity investigated in the current thalidomide's trials which minimizes its potential in the treatment of HCC. It should be noted that it is possible with the continuous development of clinical and pharmacological studies that thalidomide new derivatives become more specific and might have fewer side effects in the future and be of benefit to patients with HCC.

\section{Interferon}

Interferons (IFNs) are a group of natural cytokines that are involved in various biological processes, including anti-proliferative, antiviral and immunomodulatory actions. ${ }^{47}$ IFNs were given that name due to their ability to interfere with viral replication within living cells. ${ }^{48}$ They could be classified into 3 distinct subtypes, depending on the differences in their receptors: type I (IFN- $\alpha$ and IFN- $\beta$ ); type II (IFN- $\gamma$ ) and type III (IFN- $\lambda$ ). IFN type I is the most commonly used type for treatment. ${ }^{49}$ In general, IFN- $\alpha$ exerts its biological effect through the activation of the Janus kinease (JAK)-signal transducer and activator of transcription (STAT) signaling pathway. ${ }^{50,51}$ Upon binding of IFN- $\alpha$ to its receptor, it triggers a cascade of events leading to the activation of the transcription of IFN-stimulated genes which mediate its anti-proliferative and pro-apoptotic properties. This cascade involves the phosphorylation of IFN-receptor by JAKs which subsequently phosphorylate the STAT proteins. The activated STAT then translocates to the nucleus and activates the IFN-stimulated genes. Therefore, STAT proteins could be potential targets to enhance the efficacy of INFs therapy.

Over the past few years, accumulating evidence showed that IFN has a potent antineoplastic effect on various types of tumors, including HCC ${ }^{52-55}$ However the precise mechanism underlying this antitumor activity is still far from being clear. Like many other anti-tumor agents, the main mechanism of IFN is triggering cell apoptosis. ${ }^{54,55} \mathrm{~A}$ recent study has indicated that interferon affects hepatoma cells differently in a cell-dependent manner with a P53-independent mechanism. ${ }^{54}$ Furthermore, treating hepatoma cell lines with IFN resulted in the upregulation of promyelocitic leukemia (PML) gene expression, which is considered a tumor suppressor gene involved in mediating cell apoptosis, regardless of the P53 status. ${ }^{54}$ Furthermore, TRAIL, an IFN target gene ${ }^{56}$ is involved in cell apoptosis in IFN-treated cells in a mechanism mediated by the PML protein. ${ }^{54}$. On the other hand, knocking down PML expression using small interfering RNA (siRNA) caused a significant attenuation in the TRAIL level which subsequently halted IFNinduced apoptosis.

Moreover, IFN could act as an anti-angiogenic agent in the treatment of HCC, as it inhibits neovascularization in the tumor through the down regulation of VEGF expression which is associated with a reduction in tumor growth. ${ }^{57,58}$ The inhibition of VEGF expression could be mediated by the inhibitory effect of IFN on hypoxia-inducible factor $\alpha$ (HIF- $\alpha$ ), which is a well-known upstream activator of VEGF expression. Furthermore, a complementary DNA (cDNA) microarray analysis showed that IFN- $\alpha$ could inhibit PI3 kinase and MAP kinase pathways, which are pivotal for VEGF expression in MHCC97 cells (a metastatic HCC cell line). ${ }^{58}$

In addition to its therapeutic effect, IFN has shown to be effective in reducing the incidence of $\mathrm{HCC}$ in patients with chronic hepatitis $\mathrm{C}$ virus as well as the relapse of HCC after curative therapy or resection..$^{53}$ In contrast, there is not enough data to determine the cytotoxic effect of IFN on hepatitis B virus-related HCC. ${ }^{49}$ However, a few reports have indicated that IFN could inhibit HCC development in patients with cirrhosis resulted from the hepatitis B virus.$^{59,60}$ Very recently, Liu et al. claimed that IFN increases the sensitivity of HCC to chemotherapy through the inhibition of NF- B pathway ${ }^{61}$ which induced the expression of some anti-apoptotic agents. ${ }^{62}$

Although IFN has a modest effect on patients with advanced HCC, a considerable proportion of these patients showed resistance to IFN chemotherapy. This means that it is necessary to develop new strategies to enhance the efficacy of IFN. One of the most recent preclinical studies suggested that aspirin can significantly enhance the IFN- $\alpha$ induced apoptosis in vitro as well as tumor regression in nude mice via the JAK1/STAT1 pathway. ${ }^{9}$ Moreover, for the purpose of enhancing its cytotoxic effect, IFN was administrated to patients with advanced HCC in combination with several other therapeutic agents, such as 5Fluorouracil (5-FU), doxorubicin and cisplatin. ${ }^{6}$

In summary IFN type I is widely used in the treatment of HCC. Interestingly, IFN- $\beta$ has a greater tumoricidal effect than IFN- $\alpha$ on HCC growth, proliferation and induction of apoptosis. ${ }^{63}$ On the other hand, high doses of IFN cannot be used in patients with chronic liver diseases. Furthermore, the administration of high doses of IFN resulted in several undesirable symptoms such as weight loss, fatigue and severe hematological disorders. ${ }^{64}$

\section{Chemotherapy}

\section{Sorafenib}

Sorafenib, a tyrosine kinase inhibitor, is considered to be a standard therapy for advanced HCC patients, as it inhibits HCC growth and angiogenesis. Sorafenib was the topic of many recent reviews and therefore will not be discussed in detail here. ${ }^{65-67}$

\section{5-Fluorouracil}

5-Fluorouracil (5-FU) is one of the most widely used chemotherapeutic drugs in the treatment of several types of malignancies, such as colon, breast and HCC cancers. ${ }^{68}$ It was developed in 1950 s, as a heterocyclic aromatic compound, uracil analogue, with a fluorine substituent at the C-5 position. ${ }^{5}$ This drug can incorporate into the RNA and the DNA and interrupt the cell cycle leading to the induction of apoptosis. It is rapidly uptaken by the cell through the same transport system as uracil. ${ }^{69}$ Inside the human body, it is converted into some active metabolites that interrupt RNA and inhibit the thymidylate synthase (TS) enzyme activity, fluorodeoxyuridine monophosphate (FdUMP), fluorodeoxyuridine triphosphate FdUTP) and fluorouridine triphosphate (FUTP). Furthermore, 5-FU is catabolized into dihydrofluorouracil (DHFU) by dihydropyrimidine dehydrogenase (DPD) mainly in the liver. ${ }^{70-73}$ It performs its anticancer effect through two main mechanisms: ${ }^{74}$ inhibition of TS or misincorporation into DNA or RNA. The inhibition of the TS enzyme in turn leads to a reduction in the level of deoxythymidine monophosphate (dTMP), which ultimately causes DNA damage and cell death. ${ }^{75-77}$ The other mechanism for the cytotoxic effect of 5-FU is through the misincorporation into the nucleic acids (either DNA or RNA) instead of pyrimidine nucleotides and therefore it interferes with the normal biosynthesis and function of the nucleic acid that eventually halts cell growth. ${ }^{78}$ Indeed, 5-FU misincorporation can interrupt many aspects of RNA processing, such as inhibiting mat- 
uration of rRNA, splicing and polyadenylation of mRNA as well as disrupting the post-transcriptional modifications of the tRNA. ${ }^{78}$

In addition to these two mechanisms, Cheng et al. suggested that 5FU can induce cell apoptosis in a P53-dependent mechanism. ${ }^{79} \mathrm{He}$ indicated that 5-FU treatment was accompanied with elevated P53 expression at both the RNA and protein levels in HCC tumors. Upregulation of P53 significantly decreased the Bcl-2/Bax ratio that induced the release of cytochrome $\mathrm{C}$ from the mitochondria which in turn caused upregulation of caspase-3. Activation of caspase-3 expression triggered further apoptosis by reducing the expression of poly ADP-ribose polymerase 1 (PARP-1) at mRNA and protein levels.

Like many other anticancer agent, 5 -FU could be administrated via intraperitoneal injection, intrahepatic perfusion and intra-arterial infusion ${ }^{80}$ to enhance its therapeutic effect. It has a very short half-life in the body, therefore it should be administered in multiple doses to maintain its therapeutic blood level. Consequently, it may be associated with severe side effects, such as gastrointestinal toxicity and hematologic and bone marrow disorders. ${ }^{11}$ Therefore, a site-specific delivery system is required to provide a more effective and safer therapy. Recent studies have proposed that 5 -FU could be applied to the liver surface through a diffusion cell which ensure a site-selective delivery of the drug that minimizes systemic side effects. ${ }^{11}$ Another study used nanoparticles as delivery system to deliver 5-FU to HCC cells that ensures a controlled release of the drug to the tumor site. ${ }^{79}$ This study indicated that using 5-FU-loaded nanoparticles ensured an accumulation of the drug into the HCC tissues that caused a more potent inhibitory effect on tumor growth with lower toxicity compared with 5FU alone. These finding suggest that using nanoparticles as drug delivery system could be very promising in improving HCC chemotherapy ${ }^{79}$

\section{Cisplatin}

It was the first member of the platinum-containing anti-cancer drug class. The cytotoxic activity of the drug is its essential feature and refers to its DNA-damaging effect. Although the cisplatin cytotoxic mechanism is not fully understood, it was postulated that cisplatinDNA adducts may kill cells via apoptosis induction. ${ }^{81}$

Once cisplatin enters the cell (through transmembrane channels), the concentration of the chloride ions decreases to $20 \mathrm{mM} .^{82}$ Consequently, it is hydrated to form positively charged active species for subsequent cellular nucleophiles interaction. ${ }^{83}$ At the cellular level, cisplatin may also interact with various cellular components that have nucleophilic sites (e.g., thiol-containing molecules, proteins, RNA, membrane phospholipids, DNA) subsequently leading to cell malfunctioning and death. Furthermore, intracellular cisplatin reacts with nuclear DNA to yield DNA-protein cross-links and intrastrand DNA crosslinks. The most common is the intrastrand cross-link between adjacent guanines. ${ }^{84}$ Not only does cisplatin target genomic DNA and induce cytotoxicity, but it also targets other cellular components, which are also involved in the cytotoxicity of the drug. Therefore, cisplatin interacts with phospholipids and phosphatidylserine in membranes, binds to mitochondrial DNA, affects the polymerization of actin and disrupts the cytoskeleton. ${ }^{85}$

The mechanism of cisplatin-induced DNA damage is not the only proposed mechanism. Cisplatin cytotoxicity was observed in DNA repair-deficient cells as well, which died at cisplatin concentrations that do not inhibit DNA synthesis. Moreover, at high cisplatin concentrations, that are enough to inhibit DNA synthesis, DNA repair-proficient cells survive and block the cells in the $\mathrm{S}$ phase.$^{86}$ It means that cisplatin does not always correlate with the inhibition of DNA synthesis.

To understand the correlation between the cell death pathway and cisplatin-induced DNA damage, attention has recently been drawn to the identification and characterization of proteins that are responsible for cisplatin-induced DNA damage. It was found that more than 16 genes are essential for the DNA damage recognition and excision function of the intrastrand adduct between two adjacent guanines. ${ }^{87}$ The human mismatch repair complex hMutS-a detects but does not remove cisplatin-DNA adducts. This protein recognizes specifically a single cisplatin intrastrand adduct between two adjacent guanines..$^{88}$ There is a repair system called DNA-PK, which is mainly important for the elimination of DNA double-strand breaks that are induced by ionizing radiation. The HMG proteins are a family of small, non-histone chromatinassociated proteins involved in gene regulation and maintenance of the chromatin structure. The HMG box proteins do have the common feature of binding to DNA involved in structural deformation and some of them also bind to cisplatin-DNA adducts. ${ }^{89}$ Thus, a HMG protein called structure specific recognition protein-1 [SSRP-1] binds to cisplatin, but not transplatin adducts. ${ }^{90}$ HMG1 and HMG2 proteins recognize the intrastrand adduct between adjacent guanines. ${ }^{91}$

\section{Gemcitabine}

Gemcitabine is a fluorine-substituted deoxycytidine analog $(2$ ',2'difluorodeoxycytidine) originally developed as an antiviral agent. ${ }^{92}$ It has a broad range of anti-tumor activities against various types of solid tumors. ${ }^{93}$ Gemcitabine is also considered a pro-drug, as it undergoes intracellular modifications in order to produce its active metabolites. Once it is uptaken by the cells, it is phosphorylated either by deoxycytidine kinase (dCK) or thymidine kinase 2 to produce difluorodeoxy cytidine monophosphate (dFdCMP) which is subsequently converted to difluorodeoxy cytidine diphosphate (dFdCDP) and difluorodeoxy cytidine triphosphate (dFdCTP) active metabolites. ${ }^{92,94}$

Taking into consideration that gemcitabine specificity to $\mathrm{dCK}$ is much higher than that to thymidine kinase, the phosphorylation of gemcitabine with $\mathrm{dCK}$ is considered therefore the rate limiting step and is subsequently crucial for the cytotoxic effect. ${ }^{95} \mathrm{~A}$ deficiency of dCK could therefore be one of the potential gemcitabine drug resistance mechanisms. On the other hand, gemcitabine is inactivated rapidly by deoxycytidine deaminase (dCDA) to produce difluorodeoxyuridine (dFdU) ${ }^{96}$ Also, dFdCMP could be deaminated into 2' 2'-difluorodeoxyuridine monophosphate (dFdUMP) and ultimately to $\mathrm{dFdU}$ by the dCMP deaminase enzyme. ${ }^{93}$

Although gemcitabine is approved to be used for patients with pancreatic cancer, ${ }^{97}$ several studies demonstrated its tumoricidal effect on HCC cells. ${ }^{93}$ Preclinical studies indicated that gemcitabine had a pronounced effect on hepatoma cells in vitro. ${ }^{98}$ However, phase II trials showed that the use of gemcitabine as a single agent in treatment of advanced HCC resulted in a response rate of only 2.1-17.8\%. ${ }^{7}$ To increase its potency, a recent study indicated that using MEK inhibitors synergistically increased the sensitivity of HCC cells to gemcitabine. ${ }^{99}$ Moreover, knockdown of survivin, an antiapoptotic molecule widely expressed in several types of tumors including HCC, enhanced the cytotoxic effect of gemcitabine on HCC cells. The proposed mode of action may be based on a decrease in the level of glucose-regulated protein 78 and an induction of apoptotic signaling. ${ }^{8}$ Similarly, co-treatment of HCC cells with quinacrine sensitized these cells to various chemotherapeutic agents, including gemcitabine. This may be occur by inducing apoptosis and decreasing some anti-apoptotic proteins, such as Mcl1. ${ }^{100}$ These studies suggested that gemcitabine is recommended to be used in a combination with other therapeutic agents in order to achieve a better cytotoxic effect on cancer cells.

Despite the fact that gemcitabine has an acceptable anti-tumor agent, the precise mechanism underlying its toxicity is not yet fully understood. One of the most cited mechanisms is the competition of dFdCTP with dCTP to be incorporated into a DNA strand during DNA replication. ${ }^{101}$ Following this incorporation process, only one additional deoxynuxleotide is added to the growing DNA strand that terminates the DNA synthesis. The addition of this deoxynuxleotide protects the 
DNA chain from the DNA repair enzymes that subsequently induces cell apoptosis. ${ }^{92,102}$ Furthermore, dFdCDP active metabolite inhibits ribonucleotide reductase (RR) enzyme, which is crucial for producing deoxynucleotides (especially dCTP) needed for DNA synthesis and repair. ${ }^{103}$ The reduction of $\mathrm{dCTP}$ level favors $\mathrm{dFdCTP}$, the other gemcitabine active metabolite, for incorporation into DNA instead of dCTP in a mechanism called self-potentiation. ${ }^{104}$ In contrast, increased activity of RR elevates the dCTP pools and subsequently inhibits gemcitabine phosphorylation reducing its activity. In addition to DNA, dFdCTP could also be incorporated into RNA causing an inhibition of RNA synthesis in a time and concentration dependent manner. ${ }^{105}$ However, the precise mechanism by which gemcitabine induces cytotoxicity through incorporation into RNA is still far to be clear.

Like many other anticancer drugs, HCC cells also acquire resistance to gemcitabine leading to an insignificant improvement in terms of overall survival of HCC patients. Further studies are therefore urgently needed to uncover the molecular mechanisms underlying this resistance which may ultimately open up potential approaches leading to the identification of novel therapeutic targets for HCC.

In conclusion, gemcitabine is a promising antitumor agent in the treatment of HCC either as a single therapeutic regime or in combination with other chemotherapies.

\section{Thymophysin and $\alpha-1-$ Thymosin}

Among the experimental strategies targeting HCC, immunomodulation is considered to be a promising strategy. Likewise, immune function depression was found to play a major role in the development and pathogenesis of HCC. ${ }^{106}$ Thymostimulin, a thymic peptide fraction isolated from calf thymus including thymosin- $\alpha-1$ and thymic humoral factor, was found to be an effective immunomodulatory agent. ${ }^{107}$ It is capable of stimulating the release of interferons and interleukin- 2 and also stimulates the recovery of T-cell proliferation and differentiation in immunocompromised patients. Furthermore, it was found that administering thymostimulin to patients undergoing surgery decrease the infection incidence. Moreover, a selective dose-dependent cytotoxic immune reaction was observed against HCC cell lines in vitro. ${ }^{107-109}$ Indeed, the overall response rate was $24 \%$ and was associated with a significant increase in life expectancy in the only clinical phase II trial conducted to date. ${ }^{109} 0$ n the other hand, few side effects were observed with the use of thymostimulin. In summary, despite the full characterization of the active component(s) of thymostimulin, a tolerability profile is needed before the role of this agent can be fully defined.

\section{Capecitabine}

Capecitabine is an oral systemic pro-drug; it remains unchanged when crossing the gastrointestinal barrier and is rapidly absorbed. ${ }^{110}$ Capecitabine has been used in case of advanced HCC patients who were not eligible for surgery, local ablative therapies or chemoembolisation. Previous studies showed that capecitabine should be administered orally twice daily at a dosage of $2000 \mathrm{mg} / \mathrm{m}^{2}$ per day for 14 consecutive days and then discontinued for 7 days. This cycle has to be repeated every 21 days. The doses of capecitabine can be reduced by $25 \%$ or $50 \%$ depending on the general condition of the patient, serum bilirubin, and platelet count. Capecitabine inhibits the DNA synthesis and slows down the growth of the tumor tissue. ${ }^{110}$ At the cellular level, it is converted into 5 -FU, its only active metabolite, by thymidine phosphorylase which is present at higher levels in HCC compared to the normal healthy tissue. Accordingly, the capecitabine mode of action would be the same as 5-FU. A retrospective analysis of the efficacy and toxicity of capecitabine showed that it can be used for the treatment of HCC patients. Adverse events observed in this analysis were mostly due to HCC complications and liver. Other potential major adverse reactions observed include: cardiovascular, dermatological, hematological and hepatic effects. ${ }^{111,112}$ In conclusion, Capecitabine was found to be quite safe for the treatment of patients with HCC, including those with compensated cirrhosis. However, the objective response rate was limited.

\section{Doxorubicin}

Doxorubicin (DOX) is a metabolite of Streptomyces peucetius var. caesius that belongs to the anthracycline family. ${ }^{113}$ It was developed in the 1970s and was used as a chemotherapeutic agent in the treatment of different cancers including multiple myeloma, non-Hodgkin's and Hodgkin's lymphoma, sarcoma, lung, gastric, thyroid, ovarian, and breast cancers. ${ }^{114}$ There are several proposed mechanisms to interpret the doxorubicin antitumor activity. ${ }^{115}$ Generation of free radicals and the intercalation into DNA are considered the main cytotoxic mechanisms.

Generally, anthracyclines are well-known to intercalate into DNA in in vitro experiments. Moreover, there are many crystal structures of DNA complexes with doxorubicin in the protein data bank (e.g., pdb id 151D and 1P20). ${ }^{116}$ Previous studies showed that the intercalation of doxorubicin into DNA caused DNA breaks in addition to the interference with DNA synthesis. ${ }^{117}$ Other studies have shown that the DNAdoxorubicin interaction is related to the poisoning of topoisomerase II (TOP2A), ${ }^{18,119}$ but not topoisomerase I. ${ }^{120,121}$ Topoisomerase II-mediated DNA damage is followed by cell death. ${ }^{112}$ TP53, a gene that is a major player of the DNA-damage response and apoptosis, ${ }^{122,123}$ has been involved in this doxorubicin-apoptosis pathway. TP53 has shown to functionally interact in a p53-mediated apoptotic pathway with a doxorubicin treatment in lymphoblastoid cell lines. ${ }^{124}$

Doxorubicin can either undergo one-electron or two-electrons reduction. The first one might be mediated by microsomal NADPH P450 reductase, $\mathrm{NADH}$ cytochrome $b_{5}$ reductase or mitochondrial NADH-oxidoreductase to form the corresponding doxorubicin-semiquinone radical. ${ }^{125}$ Alternatively, a two electron reduction is mediated by $\mathrm{NAD}[\mathrm{P}] \mathrm{H}$ :quinone oxidoreductase (nqo1) (DT-diaphorase) to form the corresponding doxorubicin-hydroquinone. ${ }^{126-128}$ Re-oxidation of doxorubicin-semiquinone and doxorubicin-hydroquinone back to doxorubicin leads to the formation of reactive oxygen species (ROS) leading to oxidative stress which in turn triggers apoptosis and induces cell death. ${ }^{129-131}$

\section{Mitoxantrone}

Mitoxantrone (MX), also known as dihydroxyanthracenedione (DHAD), is a tricyclic planar chromophore with a two basic side chains. ${ }^{132}$ It exhibits an anticancer activity against various types of tumors, including HCC. ${ }^{133}$ MX was developed originally as a simplified doxorubicin analogue with a lower cardiotoxicity. ${ }^{134}$ Furthermore, it has been approved by the FDA for treatment of acute myeloid leukemia, prostate cancer and multiple sclerosis. ${ }^{134}$

Like several anticancer agents, MX inhibits cells division through intercalation into the DNA causing DNA condensation and subsequently prevents DNA replication as well as RNA synthesis. This intercalation can take place by three different modes: i) electrostatic interaction between the positively charged $\mathrm{N}$-containing side chain of MX and the negatively charged phosphate backbone DNA; ii) binding to the two grooves of the DNA double helix with a much higher affinity for the major groove than the minor groove; iii) insertion between base pairs of the DNA strand. ${ }^{132,135}$ However the precise mechanism of MX intercalation is not yet fully understood. In addition to its effect on dividing cells, MX also kills non-proliferating cells (in vitro) suggesting that its mechanism may also be a cell-cycle independent.

Treatment of HepG2 and Hep3B cell lines with MX induced cell apoptosis through accumulation of apoptotic protein such as P53, P63 and P73 inside the cells in a dose and time dependent manner. ${ }^{136} 0 \mathrm{n}$ the other hand, silencing the expression of these P53 family members 
using siRNA resulted in a significant reduction in the apoptotic cells with increased chemoresistant properties in the tumor cells. ${ }^{136}$ Furthermore, MX binds to chromatin, with a higher affinity than DNA, forming a complex that inhibits the release of histone proteins. ${ }^{137}$ Moreover, MX suppresses the topoisomerase II enzyme which is crucial for the DNA repair that leads to DNA break and induction of apoptosis. ${ }^{132}$

Clinical studies have indicated that MX is uptaken rapidly by tissues while it is eliminated in a relatively slow rate mainly by biliary excretion. ${ }^{138}$ In an early study, Stewart and colleagues used HPLC to examine the concentration of MX in different tissue samples of 11 patients who were treated with the drug intravenously 10-272 days antemortem. Interestingly, MX was detectable in tissues from all patients with the highest concentration in the thyroid and liver, while the brain had the lowest MX concentration. This finding confirms that MX remains in human tissue for a long time following the administration. ${ }^{139}$

In a preclinical study, using MX-loaded Gal-P123 nanoparticles resulted in an about 15 -fold increase in the accumulation of MX in the cells with about 2.3-fold higher toxicity than treating the cells with free MX. ${ }^{140}$ Moreover, in a recent randomized phase II trial, the median survival of HCC patients who received nanoparticles loaded by MX was increased from 3.23 months to 5.46 months in patients treated with MX injections. This enhancement is concomitant with minimal side effects, such as leukopenia and anemia. ${ }^{141}$ Interestingly, in this study, $13.6 \%$ of the patients who received MX-nanoparticles survived for 16-19 months, while all patients treated with MX injection died within the one year. This result indicated that the use of nanoparticles as a delivery system enhances the efficacy of this chemotherapeutic agent in the combat against cancer cells. ${ }^{141}$
In conclusion, MX is considered to be a promising anticancer agent in decreasing tumor progression through different potential mechanisms, including single or double strand DNA breaks and DNA-protein crosslinks. Unlike other anthraquinone derivatives, MX has a lower cardiotoxic effect probably because it does not reduce semiquinone (resulted from quinone reduction in the cardiocytes) that interacts with oxygen to produce free radicals. ${ }^{134}$

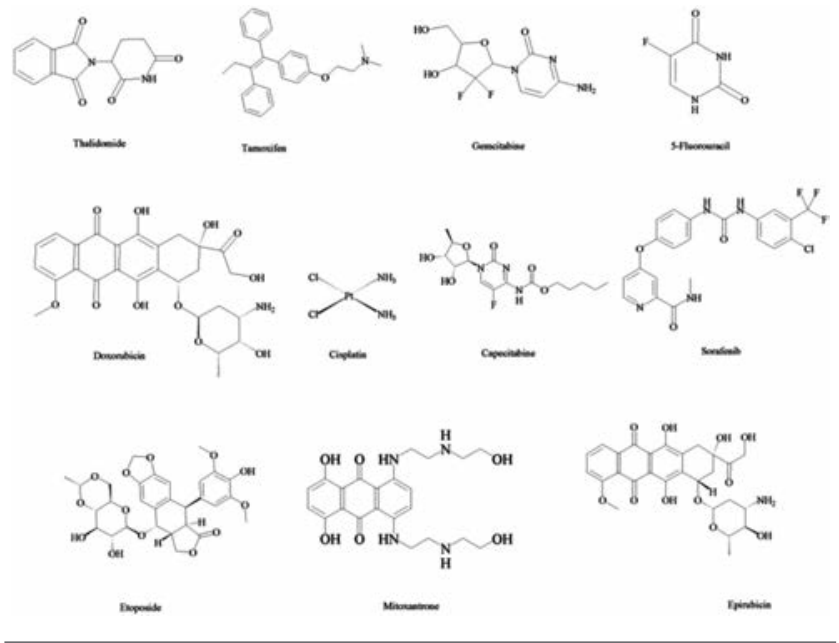

Figure 1. Chemical structures of drugs used for hepatocellular carcinoma treatment.

Table 1. Chemotherapeutic agents used for hepatocellular carcinoma treatment.

\begin{tabular}{|c|c|c|}
\hline Chemotherapeutic agent & Mode of action & References \\
\hline Octreotide & $\begin{array}{l}\text { Inhibition of growth hormone secretion, insulin and GI hormones, } \\
\text { insulin-like growth factors (IGF-I and IGF-II) and angiogenesis }\end{array}$ & $15-17,20-22$ \\
\hline Tamoxifen & $\begin{array}{l}\text { Down-regulation of mTOR activity and survivin expression } \\
\text { Blocking anti-apoptotic pathways (PI3K-Akt, and MAPK) }\end{array}$ & $23-25,27,28$ \\
\hline Thalidomide & $\begin{array}{l}\text { Down-regulation of IL-6, bFGF, VEGF, and TNF } \alpha \\
\text { Reduction of prostaglandin production } \\
\text { Anti-angiogenic agent }\end{array}$ & $35,36,42-46$ \\
\hline Interferon & $\begin{array}{l}\text { Activation of JAK-STAT signaling pathway inducing apoptosis } \\
\text { Anti-angiogenic agent }\end{array}$ & $\begin{array}{l}50,51 \\
57,58\end{array}$ \\
\hline Sorafenib & Tyrosine-kinase inhibitor & $65-67$ \\
\hline 5 -Flurouracil & $\begin{array}{l}\text { Inhibition of thymidylate synthase } \\
\text { Nucleotide analogue mis-incorporated into DNA or RNA }\end{array}$ & 71 \\
\hline Cisplatin & DNA damage & $81-84,86-89$ \\
\hline Gemcitabine & Nucleotide analogue mis-incorporated into DNA & 88,97 \\
\hline Thymophysin and $\alpha$-1-Thymosin & $\begin{array}{l}\text { Immuno-modulatory agent } \\
\text { Stimulating the release of interferons and interleukin-2 } \\
\text { Stimulation of T cell proliferation }\end{array}$ & $107-109$ \\
\hline Capecitabine & $\begin{array}{l}\text { Inhibition of DNA synthesis } \\
\text { Slowing down of tumor growth }\end{array}$ & $92-96$ \\
\hline Doxorubicin & $\begin{array}{l}\text { Generation of free radicals } \\
\text { Intercalation into DNA }\end{array}$ & $113-115,117-121$ \\
\hline Mitoxantrone & Intercalates into DNA and hence prevents DNA replication and RNA synthesis & 128,131 \\
\hline Epirubicin & Inhibition of DNA and RNA synthesis & $141-143$ \\
\hline Etoposide & Inhibitor of DNA topoisomerase II & 144-146 \\
\hline Bevacizumab & Anti-angiogenic agent & $147-150$ \\
\hline
\end{tabular}

GI, gastrointestinal; IGF, insulin-like growth factor; mTOR, mammalian target of rapamycin; MAPK, mitogen-activated protein kinase; IL-6, interleukin-6; bFGF, basic fibroblast growth factor; VEGF, vascular endothelial growth factor; TNF $\alpha$, tumor necrosis factor- $\alpha$; JAK-STAT, janus kinease-signal transducer and activator of transcription. 


\section{Epirubicin}

Epirubicin hydrochloride is a semisynthetic anthracycline cytotoxic antibiotic in which the sugar moiety differs from the natural daunosamine (amino sugar present in doxorubicin and the steric configuration of the hydroxyl bearing $\mathrm{C}-4$ is inverted, thus forming the Larabino configuration instead of the L-lyxo). Furthermore, epirubicin is considered to be a doxorubicin epimer which is only different in the spatial orientation of the hydroxyl group at the 4 carbon of the sugar moiety. This difference may account for the reduced toxicity and enhanced activity. ${ }^{142}$ In this context, data from different animal species and in vitro models have shown that epirubicin is less toxic, and in particular less cardiotoxic than doxorubicin. ${ }^{142}$ The anthracycline ring is lipophilic and the saturated end of the ring system contains hydroxyl groups adjacent to the amino sugar producing a hydrophilic centre. The molecule itself is amphoteric, including an acidic function in the phenolic ring groups and a basic function in the sugar amino group. It is mainly used as antineoplastic agent and antibiotic. The mechanism of action of epirubicin appears to be related to its ability to bind to nucleic acids, ${ }^{142}$ which leads to the complex formation due to intercalation of the planar anthracycline nucleus with the base pairs of the DNA double helix, resulting in inhibition of DNA and RNA synthesis. Intercalation also triggers DNA cleavage by topoisomerase II, resulting in cytocidal activity. ${ }^{143}$ Indeed, binding to cell membranes and plasma proteins may also be occur. Epirubicin also generates cytotoxic free radicals. ${ }^{143}$ At equally effective doses, epirubicin produces less severe non-haematologic side effects, such as vomiting and mucositis, than doxorubicin.

\section{Etoposide}

It is a semisynthetic podophyllotoxin derivative which is widely used as anticancer drug and exhibits cytotoxicity against various types of cancers. The 4 -demethylation and the introduction of a $\beta$-glycosidic moiety in position 7 of podophyllotoxin convert it into a potent irreversible inhibitor of DNA topoisomerase II. Its action is based on the formation of a nucleic acid-drug-enzyme complex, which induces breaks in single-stranded and double-stranded DNA, as the initial step in a series of biochemical transformations that eventually lead to cell death. Etoposide and its derivatives could also act through the metabolic activation of the E-ring to produce metabolites (catechol or orthoquinone) inactivating the DNA by forming chemical adducts. It was found that the microRNA (miRNA) regulates the response of the tumor cells towards chemotherapeutic agents. ${ }^{144}$ One study shed light on the potential of specific miRNA: miR-23a, which plays an important role in the regulation of the chemosensitivity of cancer cells. The overexpression of miR-23a potentiates HCC cell to etoposide-induced cell death. In miR-23a-overexpressing HCC cells, TOP2A expression remained unchanged, while TOP1 was remarkably down-regulated, leading to a fall in the overall topoisomerase activity below the critical threshold for cell survival, when cells are exposed to TOP2A poisons and consequently accelerating cell death. Both mRNA transcripts and protein expression of TOP1 are suppressed in miR-23a-overexpressing HCC cells.

Another mechanism is the activation of $\mathrm{p} 53$, which could increase the transcripts of pri-, premature and mature form of miR-23a, while inhibition of p53 significantly reduced miR-23a level in p53-proficient HCC cells, indicating that miR-23a may be transactivated by p53 in HCC cells. ${ }^{145}$ In this novel mechanism p53 was associated with Bid in the nucleus to export Bid to the mitochondria. Subsequently, in response to etoposide, it induced DNA damage in HCC which led to the induction of apoptosis. When cells were stimulated by etoposide, p53 could translocate Bid from the nucleus to the cytoplasm and on to its ultimate location in the mitochondria. P53 was physically associated with Bid, and both p53 and Bid cooperatively promoted cell death induced by etoposide. Knockdown of Bid expression notably attenuated cell death induced by etoposide and also released p53 from the mitochondria. ${ }^{146}$

\section{Molecular targeted therapy}

\section{Bevacizumab}

As claimed before, the top notable radiological and pathological characteristics of HCC besides its multicentricity are its hypervascularity and early invasion of vascular structures. The latter are mediated by the epidermal growth factor receptor (EGFR) and its ligands EGF and TGFalpha which are actively co-expressed in different human tumors, including HCC.

Bevacizumab, a recombinant humanized monoclonal antibody against VEGF, is an anti-angiogenic agent which showed to have a beneficial activity (as a single agent and in combination with chemotherapy) with response rates of up to $20 \%$ and a median survival of up to 12.4 months in HCC. ${ }^{147}$ Also, in an orthotopic HCC Hep3B model, bevacizumab significantly decreased tumor micro-vessel density, decreased human serum a-fetoprotein (AFP) and prolonged the time to progression in treatment mice compared to control mice. ${ }^{148,149}$ It has been recently accepted for the treatment of metastatic colorectal cancer, non-squamous non-small cell lung cancer, glioblastoma, metastatic breast cancer, and metastatic renal cell carcinoma. ${ }^{150}$

In addition to its direct anti-angiogenic action, bevacizumab may improve chemotherapy administration by normalizing tumor vasculature and lowering the elevated interstitial pressure in tumors. Upon binding with VEFF, bevacizumab initiates an angiogenic signal cascade and decreases tumor growth and vascularity. These in turn improve oxygenation delivery and normalize tumor vasculature. ${ }^{147}$

Bevacizumab has side effects with a grade 1 or 2 severity, such as nausea, vomiting, diarrhea, fatigue, hypertension, thrombosis and GI perforation and bleeding. The cause of GI bleeding is due to esophageal varices and/or portal hypertension. ${ }^{151}$ It is worth noting that it is advisable not to administer bevacizumab to patients who develop these symptoms.

To date, bevacizumab exact mode(s) of action is not yet fully understood. Indeed, the efficacy and safety of bevacizumab have not yet been investigated any further in phase III trials either alone or in combination with other agents for the treatment of HCC. Chemotherapeutic agents used for HCC treatment and their chemical structures are summarized in Table 115-17,20-25,27,28,35,36,42-46,50,51,57,58,65-67,71,81-84,86-89,92-97,107109,113-115,117-121,128,131,141-150 and Figure 1.

\section{References}

1. Alves RC, Alves D, Guz B, et al. Advanced hepatocellular carcinoma. Review of targeted molecular drugs. Ann Hepatol 2011; 10:21-7.

2. Bosch FX, Ribes J, Díaz M, Cléries R. Primary liver cancer: worldwide incidence and trends. Gastroenterology 2004;127:5-16.

3. Whittaker S, Marais R, Zhu AX. The role of signaling pathways in the development and treatment of hepatocellular carcinoma. Oncogene 2010:29:4989-5005.

4. Blum HE. Hepatocellular carcinoma: therapy and prevention. World J Gastroenterol 2005;11:7391-400.

5. Rutman RJ, Cantarow A, Paschkis KE. Studies in 2-acetylaminofluorene carcinogenesis. I. The intracellular distribution of nucleic acids and protein in rat liver. Cancer Res 1954;14:111-4.

6. Kaseb A0, Shindoh J, Patt YZ, et al. Modified cisplatin/interferon alpha-2b/doxorubicin/5-fluorouracil [PIAF] chemotherapy in patients with no hepatitis or cirrhosis is associated with improved response rate, resectability, and survival of initially unresectable hepatocellular carcinoma. Cancer 2013;119:3334-42.

7. Yang TS, Lin YC, Chen JS, et al. Phase II study of gemcitabine in 
patients with advanced hepatocellular carcinoma. Cancer 2000;89:750-6.

8. Hung CS, Lin SF, Liu HH, et al. Survivin-mediated therapeutic efficacy of gemcitabine through glucose-regulated protein 78 in hepatocellular carcinoma. Ann Surg Oncol 2012;19:2744-52.

9. Li T, Dong ZR, Guo ZY, et al. Aspirin enhances IFN-alpha-induced growth inhibition and apoptosis of hepatocellular carcinoma via JAK1/STAT1 pathway. Cancer Gene Ther 2013;20:366-74.

10. Song DS, Bae SH, Song MJ, et al. Hepatic arterial infusion chemotherapy in hepatocellular carcinoma with portal vein tumor thrombosis. World J Gastroenterol 2013;19:4679-88.

11. Kodama Y, Fumoto S, Nishi J, et al. Absorption and distribution characteristics of 5 -fluorouracil [5-FU] after an application to the liver surface in rats in order to reduce systemic side effects. Biol Pharm Bull 2008;31:1049-52.

12. Liu Y, Jiang L, Mu Y. Somatostatin receptor subtypes 2 and 5 are associated with better survival in operable hepatitis B-related hepatocellular carcinoma following octreotide long-acting release treatment. Oncology Lett 2013;6:821-8.

13. Marra M, Sordelli IM, Lombardi A, et al. Molecular targets and oxidative stress biomarkers in hepatocellular carcinoma: an overview. J Transl Med 2011;9:171.

14. Xi-Qing J, Xin-Jian R, Hong C, et al. Somatostatin analogues in advanced hepatocellular carcinoma: an updated systematic review and meta-analysis of randomized controlled trials. Medical science monitor: international medical J Exp Clin Res 2011;17: RA169-76.

15. Samonakis DN, Notas G, Christodoulakis N, et al. Mechanisms of action and resistance of somatostatin analogues for the treatment of hepatocellular carcinoma: a message not well taken. Digest Dis SCi 2008;53:2359-65.

16. Samonakis DN, Moschandreas J, Arnaoutis T, et al. Treatment of hepatocellular carcinoma with long acting somatostatin analogues. Oncol Rep 2002;9:903-7.

17. Li S, Liu Y, Shen Z. Characterization of somatostatin receptor 2 and 5 expression in operable hepatocellular carcinomas. Hepatogastroenterol 2012;59:2054-8.

18. Hua YP, Yin XY, Peng BG, et al. Mechanisms and influence of octreotide-induced regulation of somatostatin receptor 2 on hepatocellular carcinoma. Chemotherapy 2009;55:312-20.

19. Jia WD, Xu GL, Xu RN, et al. Octreotide acts as an antitumor angiogenesis compound and suppresses tumor growth in nude mice bearing human hepatocellular carcinoma xenografts. J Cancer Res Clin Oncol 2003;129:327-34.

20. Liu HL, Huo L, Wang L. Octreotide inhibits proliferation and induces apoptosis of hepatocellular carcinoma cells. Acta Pharmacol Sinica 2004;25:1380-6.

21. Siveke J, Folwaczny C, Herberhold C. Complete regression of advanced HCC with long acting octreotide. Gut 2003;52:1531.

22. Leung TWT, Johnson PJ. Systemic therapy for hepatocellular carcinoma. Semin Oncol 2001;28:514-20.

23. Mansour A, Daba A, Baddour N, et al. Schizophyllan inhibits the development of mammary and hepatic carcinomas induced by 7,12 dimethylbenz $[\alpha]$ anthracene and decreases cell proliferation: comparison with tamoxifen. J Cancer Res Clin Oncol 2012;138:1579-96.

24. Becker S, Ardisson V, Lepareur N, et al. Increased Lipiodol uptake in hepatocellular carcinoma possibly due to increased membrane fluidity by dexamethasone and tamoxifen. Nuclear Med Biol 2010;37:777-84.

25. Doffoël M, Bonnetain F, Bouché 0, et al. Multicentre randomised phase III trial comparing Tamoxifen alone or with Transarterial Lipiodol Chemoembolisation for unresectable hepatocellular carcinoma in cirrhotic patients [Fédération Francophone de
Cancérologie Digestive 9402]. Eur J Cancer [0xford, England: 1990] 2008;44:528-38.

26. Barbare JC, Bouché 0, Bonnetain F, et al. Randomized controlled trial of tamoxifen in advanced hepatocellular carcinoma. J Clin Oncol 2005;23:4338-46.

27. Guo R, Wang T, Shen H, et al. Involvement of mTOR and survivin inhibition in tamoxifen-induced apoptosis in human hepatoblastoma cell line HepG2. Biomed Pharmacother 2010;64:249-53.

28. Guo R, Huang Z, Shu Y, et al. Tamoxifen inhibits proliferation and induces apoptosis in human hepatocellular carcinoma cell line HepG2 via down-regulation of survivin expression. Biomed Pharmacother 2009;63:375-9.

29. Massimo DM, Bruno D, Sandro P, et al. Is human hepatocellular carcinoma a hormone-responsive tumor? World J Gastroenterol 2008;14:1682.

30. Chow PK, Tai BC, Tan CK, et al. High dose tamoxifen in the treatment of inoperable hepatocellular carcinoma: a multicenter randomized controlled trial. Hepatology 2002;36:1221-6.

31. Gallo C, De Maio E, Di Maio M, et al. Tamoxifen is not effective in good prognosis patients with hepatocellular carcinoma. BMC Cancer 2006;6:196.

32. Ang SF, Tan SH, Toh HC, et al. Activity of thalidomide and capecitabine in patients with advanced hepatocellular carcinoma. Am J Clin Oncol 2012;35:222-7.

33. Chen YY, Yen HH, Chou KC, et al. Thalidomide-based multidisciplinary treatment for patients with advanced hepatocellular carcinoma: a retrospective analysis. World J Gastroenterol 2012;18:466.

34. Feng YM, Feng CW, Chen SC, Hsu CD, et al. Unexpected remission of hepatocellular carcinoma [HCC] with lung metastasis to the combination therapy of thalidomide and cyproheptadine: report of two cases and a preliminary HCC cell line study. BMJ Case Reports $2012 ; 2012$.

35. Shao YY, Lin ZZ, Hsu C, et al. Efficacy, safety, and potential biomarkers of thalidomide plus metronomic chemotherapy for advanced hepatocellular carcinoma. Oncology 2012;82:59-66.

36. Pinter M, Wichlas M, Schmid K, et al. Thalidomide in advanced hepatocellular carcinoma as antiangiogenic treatment approach: a phase I/II trial. Eur J Gastroenterol Hepatol 2008;20:1012-9.

37. Takuji T, Hideki I, Toru N, et al. Metronomic chemotherapy: possible clinical application in advanced hepatocellular carcinoma. Transl Oncol 2013;6:511.

38. Ali A, Hothi SS, Thompson A, et al. Negative chronotropic effects and coronary ischaemic abnormalities following thalidomide therapy. Cardiology 2013;125:34-37.

39. Kawamura Y, Shirotsuka Y, Awatsuji H, et al. Common nature in the effects of thalidomide on embryo-fetal development in Kbl:JW and Kbl:NZW rabbits. Congenital Anomalies 2013;54:41-53.

40. Jasielec JK, Jakubowiak AJ. Current approaches to the initial treatment of symptomatic multiple myeloma. Int J 2013;2:61-70.

41. Morabito F, Bringhen S, Larocca A, et al. Bortezomib, Melphalan, Prednisone [VMP] versus Melphalan, Prednisone, Thalidomide [MPT] in elderly newly diagnosed Multiple Myeloma patients: a retrospective case matched study. Am J Hematol 2013;89:355-62.

42. Hsu C, Chen CN, Chen LT, et al. Low-dose thalidomide treatment for advanced hepatocellular carcinoma. Oncology 2003;65:242-49.

43. Chen YY, Yen HH, Chou KC, et al. Thalidomide in the treatment of patients with hepatocellular carcinoma. Cancer 2005;103:749-55.

44. Yau T, Chan P, Wong H, et al. Efficacy and tolerability of low-dose thalidomide as first-line systemic treatment of patients with advanced hepatocellular carcinoma. Oncology 2007;72:67-71.

45. Chiou HE, Wang TE, Wang YY, et al. Efficacy and safety of thalidomide in patients with hepatocellular carcinoma. World $\mathrm{J}$ Gastroenterol 2006;12:6955. 
46. Zhang ZI, Liu ZS, Sun Q. Anti-tumor effect of thalidomide and paclitaxel on hepatocellular carcinoma in nude mice. Chinese Med J 2005;118:1688.

47. George PM, Badiger R, Alazawi W, et al. Pharmacology and therapeutic potential of interferons. Pharmacol Ther 2012;135:44-53.

48. Hayashi N, Takehara T, Antiviral therapy for chronic hepatitis C: past, present, and future. J Gastroenterol 2006;41:17-27.

49. Zhuang L, Zeng X, Yang Z, Meng Z, et al. Effect and safety of interferon for hepatocellular carcinoma: a systematic review and metaanalysis. PLoS One 2013;8:e61361.

50. Darnell JJ, Kerr IM, Stark GR. Jak-STAT pathways and transcriptional activation in response to IFNs and other extracellular signaling proteins. Science 1994;264:1415-21.

51. Ihle JN, Witthuhn BA, Quelle FW, et al. Signaling by the cytokine receptor superfamily: JAKs and STATs. Trends Biochem Sci 1994;19:222-7.

52. Shiratori Y, Ito Y, Yokosuka 0, et al. Antiviral therapy for cirrhotic hepatitis C: association with reduced hepatocellular carcinoma development and improved survival. Ann Intern Med 2005;142:105-14.

53. Nishiguchi S, Shiomi S, Nakatani S, et al. Prevention of hepatocellular carcinoma in patients with chronic active hepatitis $\mathrm{C}$ and cirrhosis. Lancet 2001;357:196-7.

54. Herzer K, Hofmann TG, Teufel A, et al. IFN-alpha-induced apoptosis in hepatocellular carcinoma involves promyelocytic leukemia protein and TRAIL independently of p53. Cancer Res 2009;69:855-62.

55. Zhang T, Sun HC, Zhou HY, et al. Interferon alpha inhibits hepatocellular carcinoma growth through inducing apoptosis and interfering with adhesion of tumor endothelial cells. Cancer Lett 2010;290:204-10.

56. Chawla SM, Lindner DJ, Liu YF, et al. Apoptosis and interferons: role of interferon-stimulated genes as mediators of apoptosis. Apoptosis 2003;8:237-49.

57. Albini A, Marchisone C, Del Grosso F, et al. Inhibition of angiogenesis and vascular tumor growth by interferon-producing cells: a gene therapy approach. Am J Pathol 2000;156:1381-93.

58. Wu WZ, Sun HC, Shen YF, et al. Interferon alpha 2a down-regulates VEGF expression through PI3 kinase and MAP kinase signaling pathways. J Cancer Res Clin Oncol 2005;131:169-78.

59. Ikeda K, Saitoh S, Suzuki Y, et al. Interferon decreases hepatocellular carcinogenesis in patients with cirrhosis caused by the hepatitis B virus: a pilot study. Cancer 1998;82:827-35.

60. Yang JQ, Pan GD, Chu GP, et al. Interferon-alpha restrains growth and invasive potential of hepatocellular carcinoma induced by hepatitis B virus X protein. World J Gastroenterol 2008;14:5564-9.

61. Liu Y, Lou G, Wu W, Shi Y, et al. Interferon-alpha sensitizes HBxexpressing hepatocarcinoma cells to chemotherapeutic drugs through inhibition of HBx-mediated NF-kappaB activation. Virol J 2013;10:168.

62. Nakanishi C, Toi M, Nuclear factor-kappaB inhibitors as sensitizers to anticancer drugs. Nat Rev Cancer 2005;5:297-309.

63. Murata M, Nabeshima S, Kikuchi K, et al. A comparison of the antitumor effects of interferon-alpha and beta on human hepatocellular carcinoma cell lines. Cytokine 2006;33:121-8.

64. Wang L, Tang ZY, Qin LX, et al. High-dose and long-term therapy with interferon-alfa inhibits tumor growth and recurrence in nude mice bearing human hepatocellular carcinoma xenografts with high metastatic potential. Hepatology 2000;32:43-8.

65. Shen YC, Lin ZZ, Hsu CH, et al. Clinical trials in hepatocellular carcinoma: an update. Liver Cancer 2013;2:345-64.

66. Welker MW, Trojan J. Antiangiogenic treatment in hepatocellular carcinoma: the balance of efficacy and safety. Cancer Manag Res 2013;5:337-47.
67. Finn RS. Current and future treatment strategies for patients with advanced hepatocellular carcinoma: role of mTOR inhibition. Liver Cancer 2012;1:247-56.

68. Grem JL. 5-Fluorouracil: forty-plus and still ticking. A review of its preclinical and clinical development. Invest New Drugs 2000;18:299-13.

69. Wohlhueter RM, McIvor RS, Plagemann PG, Facilitated transport of uracil and 5-fluorouracil, and permeation of orotic acid into cultured mammalian cells. J Cell Physiol 1980;104:309-19.

70. He YF, Wei W, Zhang X, et al. Analysis of the DPYD gene implicated in 5-fluorouracil catabolism in Chinese cancer patients. J Clin Pharm Ther 2008;33:307-14.

71. Longley DB, Latif T, Boyer J, et al. The interaction of thymidylate synthase expression with p53-regulated signaling pathways in tumor cells. Semin Oncol 2003;30:3-9.

72. Parker WB, Cheng YC. Metabolism and mechanism of action of 5fluorouracil. Pharmacol Ther 1990;48:381-95.

73. Zhang $\mathrm{N}$, Yin $\mathrm{Y}, \mathrm{Xu}$ SJ, et al. 5-Fluorouracil: mechanisms of resistance and reversal strategies. Molecules 2008;13:1551-69.

74. Kojiro S, Yano H, Ogasawara S, et al. Antiproliferative effects of 5fluorouracil and interferon-alpha in combination on a hepatocellular carcinoma cell line in vitro and in vivo. J Gastroenterol Hepatol 2006;21:129-37.

75. Houghton JA, Tillman DM, Harwood FG, Ratio of 2'-deoxyadenosine-5'-triphosphate/thymidine-5'-triphosphate influences the commitment of human colon carcinoma cells to thymineless death. Clin Cancer Res 1995;1:723-30.

76. Yoshioka A, Tanaka S, Hiraoka 0, et al. Deoxyribonucleoside triphosphate imbalance. 5-Fluorodeoxyuridine-induced DNA double strand breaks in mouse FM3A cells and the mechanism of cell death. J Biol Chem 1987;262:8235-41.

77. Longley DB, Harkin DP, Johnston PG, 5-fluorouracil: mechanisms of action and clinical strategies. Nat Rev Cancer 2003;3:330-8.

78. Noordhuis P, Holwerda U, Van der Wilt CL, et al. 5-Fluorouracil incorporation into RNA and DNA in relation to thymidylate synthase inhibition of human colorectal cancers. Ann Oncol 2004;15:1025-32.

79. Cheng M, Mail BH, Wan T, et al. 5-Fluorouracil nanoparticles inhibit hepatocellular carcinoma via activation of the p53 pathway in the orthotopic transplant mouse model. PLoS One 2012;7:e47115.

80. van der Wilt CL, Marinelli A, Pinedo HM, et al. The effect of different routes of administration of 5 -fluorouracil on thymidylate synthase inhibition in the rat. Eur J Cancer 1995;31:754-60.

81. Eastman A. The mechanism of action of cisplatin: from adducts to apoptosis. In: Lippert B, ed. Cisplatin: chemistry and biochemistry of a leading anticancer drug. Zürich: Verlag Helvetica Chimica Acta; 2006.

82. Gately DP, Howell SB. Cellular accumulation of the anticancer agent cisplatin: a review. Br J Cancer 1993;67:1171-6.

83. Andrews PA, Howell SB. Cellular pharmacology of cisplatin: perspectives on mechanisms of acquired resistance. Cancer Cells 1990;2:35-43.

84. Perez RP. Cellular and molecular determinants of cisplatin resistance. Eur J Cancer 1998;34:1535-42.

85. Jamieson ER, Lippard SJ. Structure, recognition, and processing of cisplatin-DNA adducts. Chem Rev 1999;99:2467-98.

86. Sorenson CM, Eastman A. Influence of cis-diamminedichloroplatinum[II] on DNA synthesis and cell cycle progression in excision repair proficient and deficient Chinese hamster ovary cells. Cancer Res 1988;48:6703-7.

87. Mu D, Hsu DS, Sancar A. Reaction mechanism of human DNA repair excision nuclease. J Biol Chem 1996;27:8285-94.

88. Yamada M, O'Regan E, Brown R, Karran P. Selective recognition of 
a cisplatin-DNA adduct by human mismatch repair proteins. Nucleic Acids Res 1997;25:491-6.

89. Zamble DB, Lippard SJ. The response of cellular proteins to cisplatin-damaged DNA. In: Lippert B, ed. Cisplatin: chemistry and biochemistry of a leading anticancer drug. Zürich: Verlag Helvetica Chimica Acta; 2006.

90. Bruhn SL, Pil PM, Essigmann JM, Housman DE. Isolation and characterization of human cDNA clones encoding a high mobility group box protein that recognizes structural distortions to DNA caused by binding of the anticancer agent cisplatin. Proc Natl Acad Sci U S A 1992;89:2307-11.

91. Hughes EN, Engelsberg BN, Billings PC. Purification of nuclear proteins that bind to cisplatin-damaged DNA. Identity with high mobility group proteins 1 and 2. J Biol Chem 1992;267:13520-7.

92. Mini E, Nobili S, Caciagli B, et al. Cellular pharmacology of gemcitabine. Ann Oncol 2006;17:v7-12.

93. Venook AP, Egorin MJ, Rosner GL, et al, Phase I and pharmacokinetic trial of gemcitabine in patients with hepatic or renal dysfunction: Cancer and Leukemia Group B 9565. J Clin Oncol 2000;18:2780-7.

94. Heinemann V, Hertel LW, Grindey GB, Plunkett W. Comparison of the cellular pharmacokinetics and toxicity of 2',2'-difluorodeoxycytidine and 1-beta-D-arabinofuranosylcytosine. Cancer Res 1988;48:4024-31.

95. Wang L, Munch PB, Herrström SA, et al. Human thymidine kinase 2: molecular cloning and characterisation of the enzyme activity with antiviral and cytostatic nucleoside substrates. FEBS Lett 1999;443:170-4.

96. Heinemann V, Xu YZ, Chubb S, et al. Cellular elimination of 2',2'difluorodeoxycytidine 5'-triphosphate: a mechanism of self-potentiation. Cancer Res 1992;52:533-9.

97. Burris HA 3rd, Moore MJ, Andersen J, et al. Improvements in survival and clinical benefit with gemcitabine as first-line therapy for patients with advanced pancreas cancer: a randomized trial. J Clin Oncol 1997;15:2403-13.

98. Graziadei I, Kelly T, Schirmer M, et al. Antitumor effect of the nucleoside analogs 2-chlorodeoxyadenosine and 2',2'-difluorodeoxycytidine on human hepatoma HepG2 cells. J Hepatol 1998;28:504-9.

99. Lin S, Hoffmann K, Xiao Z, et al. MEK inhibition induced downregulation of MRP1 and MRP3 expression in experimental hepatocellular carcinoma. Cancer Cell Int 2013;13:3.

100. Wang W, Gallant JN, Katz SI, et al. Quinacrine sensitizes hepatocellular carcinoma cells to TRAIL and chemotherapeutic agents. Cancer Biol Ther 2011;12:229-38.

101. Huang P, Chubb S, Hertel LW, et al. Action of 2',2'-difluorodeoxycytidine on DNA synthesis. Cancer Res 1991;51:6110-7.

102. Gandhi V, Legha J, Chen F, et al. Excision of 2',2'-difluorodeoxycytidine [gemcitabine] monophosphate residues from DNA. Cancer Res 1996;56:4453-9.

103. Heinemann V, Xu YZ, Chubb S, et al. Inhibition of ribonucleotide reduction in CCRF-CEM cells by 2',2'-difluorodeoxycytidine. Mol Pharmacol 1990;38:567-72.

104. Plunkett W, Huang P, Gandhi V. Preclinical characteristics of gemcitabine. Anticancer Drugs 1995;6:7-13.

105. Ruiz van Haperen VW, Veerman G, Vermorken JB, et al. 2',2'Difluoro-deoxycytidine [gemcitabine] incorporation into RNA and DNA of tumour cell lines. Biochem Pharmacol 1993;46:762-6.

106. Palmieri G, Biondi E, Morabito A, et al. Thymostimulin treatment of hepatocellular carcinoma on liver cirrhosis. Int J Oncol 1996;8:827-32.

107. Dollinger MM, Lautenschlaeger C, Lesske J, et al. Thymostimulin versus placebo for palliative treatment of locally advanced or metastasised hepatocellular carcinoma: a phase III clinical trial. BMC Cancer 2010;10:457.
108. Dollinger MM, Lautenschlaeger C, Lesske J, et al. Thymostimulin in advanced hepatocellular carcinoma: a phase II trial. BMC Cancer 2008;8:72.

109. Dollinger MM, Lautenschlaeger C, Lesske J, et al. Thymostimulin in advanced hepatocellular carcinoma: a phase II trial. BMC Cancer 2008;8:1-8.

110. Walko CM, Lindley C. Capecitabine: a review. Clin Ther 2005;27:23-44.

111. Wong M, Choo SP, Tan EH. Travel warning with capecitabine. Ann Oncol 2009;20:1281.

112. Baird R, Biondo A, Chhaya V, et al. Toxicity associated with capecitabine plus oxaliplatin in colorectal cancer before and after an institutional policy of capecitabine dose reduction. Br J Cancer 2011;104:43-50.

113. Arcamone F, Cassinelli G, Fantini G, et al. Adriamycin, 14-hydroxydaunomycin, a new antitumor antibiotic from S. peucetius var. caesius. Biotechnol Bioeng 1969;11:1101-10.

114. Weiss RB. The anthracyclines: will we ever find a better doxorubicin? Semin Oncol 1992;19:670-86.

115. Gewirtz DA. A critical evaluation of the mechanisms of action proposed for the antitumor effects of the anthracycline antibiotics adriamycin and daunorubicin. Biochem Pharmacol 1999;57:727-41.

116. Lipscomb LA, Peek ME, Zhou FX, et al. Water ring structure at DNA interfaces: hydration and dynamics of DNA-anthracycline complexes. Biochemistry 1994;33:3649-59.

117. Zunina F, Gambetta R, Di Marco A. The inhibition in vitro of DNA polymerase and RNA polymerases by daunomycin and adriamycin. Biochem Pharmacol 1975;24:309-11.

118. Bodley A, Liu LF, Israel M, et al. DNA topoisomerase II-mediated interaction of doxorubicin and daunorubicin congeners with DNA. Cancer Res 1989;49:5969-78.

119. Tewey KM, Rowe TC, Yang L, et al. Adriamycin-induced DNA damage mediated by mammalian DNA topoisomerase II. Science 1984;226:466-8.

120. Wassermann K, Markovits J, Jaxel C, et al. Effects of morpholinyl doxorubicins, doxorubicin, and actinomycin D on mammalian DNA topoisomerases I and II. Mol Pharmacol 1990;38:38-45.

121. Guano F, Pourquier P, Tinelli S, et al. Topoisomerase poisoning activity of novel disaccharide anthracyclines. Mol Pharmacol 1999;56:77-84.

122. Perego P, Corna E, De Cesare M, et al. Role of apoptosis and apoptosis-related genes in cellular response and antitumor efficacy of anthracyclines. Curr Med Chem 2001;8:31-7.

123. Hickman ES, Moroni MC, Helin K. The role of p53 and pRB in apoptosis and cancer. Curr Opin Genet Dev 2002;12:60-6.

124. Robles AI, Wang XW, Harris CC. Drug-induced apoptosis is delayed and reduced in XPD lymphoblastoid cell lines: possible role of TFIIH in p53-mediated apoptotic cell death. Oncogene 1999;18:4681-8.

125. Minotti G. Reactions of adriamycin with microsomal iron and lipids. Free Radic Res Commun 1989;7:143-8.

126. Pawłowska J, Tarasiuk J, Wolf CR, et al. Differential ability of cytostatics from anthraquinone group to generate free radicals in three enzymatic systems: NADH dehydrogenase, NADPH cytochrome $\mathrm{P} 450$ reductase, and xanthine oxidase. Oncol Res 2003;13:245-52.

127. Nohl H, Gille L, Staniek K. The exogenous NADH dehydrogenase of heart mitochondria is the key enzyme responsible for selective cardiotoxicity of anthracyclines. Z Naturforsch C 1998;53:279-85.

128. Vásquez-Vivar J, Martasek P, Hogg N, et a. Endothelial nitric oxide synthase-dependent superoxide generation from adriamycin. Biochemistry 1997;36:11293-7.

129. Minotti G, Recalcati S, Mordente A, et al. The secondary alcohol 
metabolite of doxorubicin irreversibly inactivates aconitase/iron regulatory protein-1 in cytosolic fractions from human myocardium. FASEB J 1998;12:541-52.

130. Miyamoto Y, Koh YH, Park YS, et al. Oxidative stress caused by inactivation of glutathione peroxidase and adaptive responses. Biol Chem 2003;384:567-74.

131. Wang S, Kotamraju S, Konorev E, et al. Activation of nuclear factor-kappaB during doxorubicin-induced apoptosis in endothelial cells and myocytes is pro-apoptotic: the role of hydrogen peroxide. Biochem J 2002;367:729-40.

132. Varadwaj P, Misra K, Sharma A, et al. Mitoxantrone: an agent with promises for anticancer therapies. Electronic J Biol 2010;6:36-42.

133. Shenkenberg TD, Von Hoff DD. Mitoxantrone: a new anticancer drug with significant clinical activity. Ann Intern Med 1986;105:67-81.

134. Fox EJ. Mechanism of action of mitoxantrone. Neurology 2004;63:15-8.

135. Xia C, Guoli S, Jianhui J, Ruqin Y. Intercalation of pharmorubicin anticancer drug to DNA studied by cyclic voltammetry with analytical applications. Anal Lett 1999;32:717-27.

136. Seitz SJ, Schleithoff ES, Koch A, et al. Chemotherapy-induced apoptosis in hepatocellular carcinoma involves the p53 family and is mediated via the extrinsic and the intrinsic pathway. Int $\mathrm{J}$ Cancer 2010;126:2049-66.

137. Hajihassan Z, Chadegani AR. Studies on the binding affinity of anticancer drug mitoxantrone to chromatin, DNA and histone proteins. J Biomed Sci 2009;16:31.

138. Ehninger G, Schuler U, Proksch B, et al. Pharmacokinetics and metabolism of mitoxantrone. A review. Clin Pharmacokinet 1990;18:365-80.

139. Stewart DJ, Green RM, Mikhael NZ, et al. Human autopsy tissue concentrations of mitoxantrone. Cancer Treat Rep 1986;70:1255-61.

140. Zhang X, Guo S, Fan R, et al. Dual-functional liposome for tumor targeting and overcoming multidrug resistance in hepatocellular carcinoma cells. Biomaterials 2012;33:7103-14.

141. Zhou Q, Sun X, Zeng L, et al. A randomized multicenter phase II clinical trial of mitoxantrone-loaded nanoparticles in the treatment of 108 patients with unresected hepatocellular carcinoma. Nanomedicine 2009;5:419-23.

142. Pfizer, PHARMORUBICIN® product monograph. Kirkland, Quebec; 2005.

143. Pfizer, ELLENCE® product monograph. New York; 2005.

144. Xia L, Zhang D, Du R, et al. miR-15b and miR-16 modulate multidrug resistance by targeting BCL2 in human gastric cancer cells. Int J Cancer 2008;123:372-9.

145. Wang N, Zhu M, Tsao SW, et al. MiR-23a-mediated inhibition of topoisomerase 1 expression potentiates cell response to etoposide in human hepatocellular carcinoma. Mol Cancer 2013;12:119.

146. Song G, Chen GG, Yun JP, Lai PB. Association of p53 with Bid induces cell death in response to etoposide treatment in hepatocellular carcinoma. Curr Cancer Drug Targets 2009;9:871-80.

147. Fang P, Hu JH, Cheng ZG, et al. Efficacy and safety of bevacizumab for the treatment of advanced hepatocellular carcinoma: a systematic review of phase II trials. PLoS ONE 2012;7:e49717.

148. Finn RS, Bentley G, Britten CD, et al. Targeting vascular endothelial growth factor with the monoclonal antibody bevacizumab inhibits human hepatocellular carcinoma cells growing in an orthotopic mouse model. Liver Int 2009;29:284-90.

149. Ong LC, Song IC, Jin Y, et al. Effective inhibition of xenografts of hepatocellular carcinoma [HepG2] by rapamycin and bevacizumab in an intrahepatic model. Mol Imaging Biol 2009;11:334-42.

150. Zustovich F, Ferro A, Lombardi G, et al. Bevacizumab as front-line treatment of brain metastases from solid tumors: a case series. Anticancer Res 2013;33:4061-5.

151. Siegel AB, Cohen EI, Ocean A, et al. Phase II trial evaluating the clinical and biologic effects of bevacizumab in unresectable hepatocellular carcinoma. J Clin Oncol 2008;26:2992-8. 